Background: Filgotinib is an orally administered small molecule that provides selective inhibition of JAK1, a signaling molecule that helps drive inflammatory pathways underlying rheumatoid arthritis (RA)

Objectives: Exposure-response (ER) analyses were performed for efficacy following completion of Phase 2 studies over a wide range of doses to support evaluation of $200 \mathrm{mg}$ and $100 \mathrm{mg}$ once daily in Phase 3 studies. ER analyses were subsequently performed by using Phase 3 efficacy data to support selection of the proposed registrational dose. ER analyses for safety based on pooled Phase 2 and Phase 3 studies were conducted to examine the safety of evaluated doses. Methods: Population PK analyses were conducted to estimate plasma exposures of filgotinib and GS-829845 (major circulating active metabolite of filgotinib) in both Phase 2 (DARWIN 1 and DARWIN 2) and Phase 3 studies (FINCH 1, FINCH 2, and FINCH 3) encompassing a dose range of 25 to $100 \mathrm{mg}$ twice daily and 50 to $200 \mathrm{mg}$ once daily. As both filgotinib and GS-829845 contribute to efficacy via JAK1 inhibition, their exposures were combined into single parameters, AUCeff and Ctau-eff (effective area under the curve and effective concentration at trough, by accounting for relative inhibition potency and molecular weight) in the ER analyses for various efficacy endpoints (e.g ACR20/50/70 responses) at Week 12 and Week 24. The ER analyses for safety endpoints (the 5 most frequent treatment-emergent adverse events [TEAEs] and Grade 3 or 4 laboratory abnormalities, serious TEAEs, and serious infections) were performed separately for filgotinib and GS-829845 exposures to characterize the individual safety profile of each analyte. The 5 evaluated TEAEs were nausea, nasopharyngitis, upper respiratory tract infection, headache, and hypertension; the 5 Grade 3/4 laboratory abnormalities included lymphocytes decrease, glucose increase, phosphate decrease, triacylglycerol lipase increase, and creatine kinase increase.

Results: In the ER analyses for efficacy based on Phase 2 studies, high response rates were demonstrated in ACR20/50/70 across all octile groups in subjects with RA receiving filgotinib and the ER supported further evaluation of both $200 \mathrm{mg}$ and $100 \mathrm{mg}$ once daily doses in Phase 3 clinical studies. Similarly, ER relationships based on pooled Phase 3 studies across various endpoints (e.g ACR20/50/70) consistently revealed high response rates across the exposure range for both the filgotinib $200 \mathrm{mg}$ and $100 \mathrm{mg}$ doses. A trend of increasing response with increasing exposure was observed over the exposure range for multiple secondary efficacy endpoints including ACR50 and ACR70 with the effective exposures at filgotinib $200 \mathrm{mg}$ primarily residing on the plateau of the ER curves.

Filgotinib was generally well-tolerated with no individual TEAE or Grade 3 or 4 laboratory abnormality $>5 \%$ in the filgotinib $200 \mathrm{mg}$ once daily group up to Week 12. No relationships were observed between filgotinib and GS-829845 exposures (AUC0-24 and Cmax) and the most frequent TEAEs, Grade 3/4 laboratory abnormalities, serious TEAEs, or serious infections up to Week 52.

Conclusion: ER analyses demonstrate that both the $200 \mathrm{mg}$ and $100 \mathrm{mg}$ once daily filgotinib doses are efficacious in subjects with moderately to severely active RA without clear dose-dependent effects on safety. The trend towards greater efficacy with higher exposures for some secondary endpoints (ACR50 and ACR70) and a lack of exposure-safety relationship supports a dose of $200 \mathrm{mg}$ once daily over $100 \mathrm{mg}$ once daily since it presents the best benefit/risk ratio among the doses tested.

Disclosure of Interests: : Amy Meng Shareholder of: Gilead Sciences, Employee of: Gilead, Kacey Anderson Shareholder of: Gilead Sciences, Employee of: Sciences, Cara Nelson Shareholder of: Gilead, Employee of: Gilead, Brian Kirby Shareholder of: Gilead, Employee of: Gilead, Liyun Ni Shareholder of: Gilead, Employee of: Gilead, Shu-Min Chuang Shareholder of: Gilead, Employee of: Gilead, Brian Kearney Shareholder of: Gilead, Employee of: Gilead, Anita Mathias Shareholder of: Gilead, Employee of: Gilead DOI: 10.1136/annrheumdis-2020-eular.4977

\section{SAT0150 \\ CHANGES IN PATIENT-REPORTED OUTCOME (PRO) SCORES FOR NAUSEA AND FATIGUE FOLLOWING WEEKLY METHOTREXATE DOSE IN A REAL- WORLD SAMPLE OF RA AND PSA PATIENTS INTHE ARTHRITISPOWER REGISTRY}

W. B. Nowell ${ }^{1}$, E. Karis ${ }^{2}$, K. Gavigan ${ }^{1}$, L. Stradford ${ }^{1}$, S. Stryker ${ }^{2}$, H. Yun ${ }^{3}$, S. Venkatachalam ${ }^{1}$, G. Kricorian ${ }^{2}$, L. Chen ${ }^{3}$, H. Zhao ${ }^{3}$, F. Xie $^{3}$, J. Curtis $^{3}$. ${ }^{1}$ Global Healthy Living Foundation, Upper Nyack, United States of America; ${ }^{2}$ Amgen Inc., Thousand Oaks, United States of America; ${ }^{3}$ University of Alabama at Birmingham, Birmingham, United States of America

Background: Methotrexate (MTX) is frequently used in patients with rheumatoid arthritis (RA) or psoriatic arthritis (PsA) because of its beneficial effects in both populations ${ }^{1-3}$. Despite the well-known benefits of MTX, it is associated with a number of potential side effects ${ }^{4-6}$ These include nausea and fatigue, are often temporally related to the timing of weekly MTX administration, and can be severe. The combined patient-reported side effects, along with potential of long-term toxicity, may make use of MTX more burdensome. Currently, there is a gap in patient-centered studies that focus on patients' experience with MTX.

Objectives: Examine patient temporal experience of fatigue and nausea relating to oral MTX therapy for the treatment of RA and PsA

Methods: Adult US patients in the ArthritisPower registry with self-reported RA or PsA taking MTX for less than 10 years were invited to participate in the study via email invitation. Participants (pts) completed a screener and brief online survey. In an ancillary study to the ArthritisPower registry and using a self-controlled case series study design where pts serve as their own control to avoid between-person confounding, pts were asked to complete a set of up to 8 assessments within 6-36 hours ('risk') and 96-144 hours ('control') after taking their oral dose of MTX each week, for up to 4 weeks. Risk and control windows were selected based on the expected temporal relationship between MTX use and peak onset of these symptoms. Assessments included PROMIS short forms for same-day Fatigue, same-day Nausea/Vomiting, and Patient Global. Descriptive statistics were conducted using paired t-tests two-way comparisons. Within-person change in PROMIS scores between the risk (1-2 days after MTX) and control (4-6 days after MTX) windows were analyzed using mixed models for repeated measures, stratified on whether pts reported fatigue or nausea with MTX at baseline. Recruitment for this study is ongoing.

Results: As of December 2019, 91 pts had participated, of whom 76.9\% were living with RA and $28.6 \%$ with PsA, with mean baseline PROMIS Patient Global score (SD) of 39.5 (7.1). Mean age (SD) was 50.9 (12.0) years, $84.6 \%$ female, $92.3 \%$ White, with mean BMI 33.7 (8.8). Mean duration of MTX treatment among current users was 2.1 (2.8) years. Among pts, $41.8 \%$ were on a biologic DMARD and $58.2 \%$ on non-biologic DMARDs only. Among pts reporting baseline nausea $(n=30,33.0 \%)$ where paired within-week measures were observed $(n=64$ observations among 20 pts), the mean increase in the PROMIS Nausea score was 4.5 units (adjusted $p=0.003$ ). Among those reporting MTX-associated fatigue $(n=39$ $42.9 \%$ ) as a side effect of MTX on their baseline survey where paired within-week measures were observed ( $n=96$ observations among 28 pts), the mean increase in PROMIS Fatigue was 4.7 (adjusted $p=0.004$ ) units. In those pts, the proportion of pts with worsened nausea and fatigue with minimally important difference of $>5$ units $^{7-8}$ was $40.0 \%$ (nausea), and $60.7 \%$ (fatigue) [Figures 1 and 2].

Conclusion: People taking MTX to manage RA or PSA commonly experience bothersome side effects, notably fatigue and nausea, that are temporally related to weekly MTX dosing. In this sample, one-third or more of pts were bothered by nausea or fatigue shortly after MTX dosing, many of them with clinically meaningful symptoms.

References:

[1] Singh JA, et al. Arthritis Rheumatol. 2016;68:1-26.

[2] Singh JA, et al. Arthritis Rheumatol. 2019;71:5-32.

[3] Mease P. Bull NYU Hosp Jt Dis. 2013;71.(suppl 1):S41.

[4] Wang W, et al. Eur J Med Chem. 2018;158:502-516.

[5] Wilsdon TD, et al. Cochrane Database Syst Rev. 2019;1:CD012722.

[6] Husted JA, et al. Ann Rheum Dis. 2009;68:1553-1558.

[7] Norman GR, et al. Med Care. 2003;41:582-92.

[8] Bingham CO, et al. J Patient Rep Outcomes. 2019;3:14.

Disclosure of Interests: W. Benjamin Nowell: None declared, Elaine Karis Shareholder of: Amgen Inc., Employee of: Amgen Inc., Kelly Gavigan: None declared, Laura Stradford: None declared, Scott Stryker Shareholder of: Amgen Inc., Employee of: Amgen Inc., Huifeng Yun Grant/research support from: Bristol-Myers Squibb and Pfizer, Shilpa Venkatachalam: None declared, Greg Kricorian Shareholder of: Amgen Inc., Employee of: Amgen Inc., Lang Chen:

Figure 1. Nausea PROMIS Score Changes Among Patients Reporting Nausea as a Side Effect of MTX [ $n=64$ observations ( 32 pairs) among 20 unique pts]

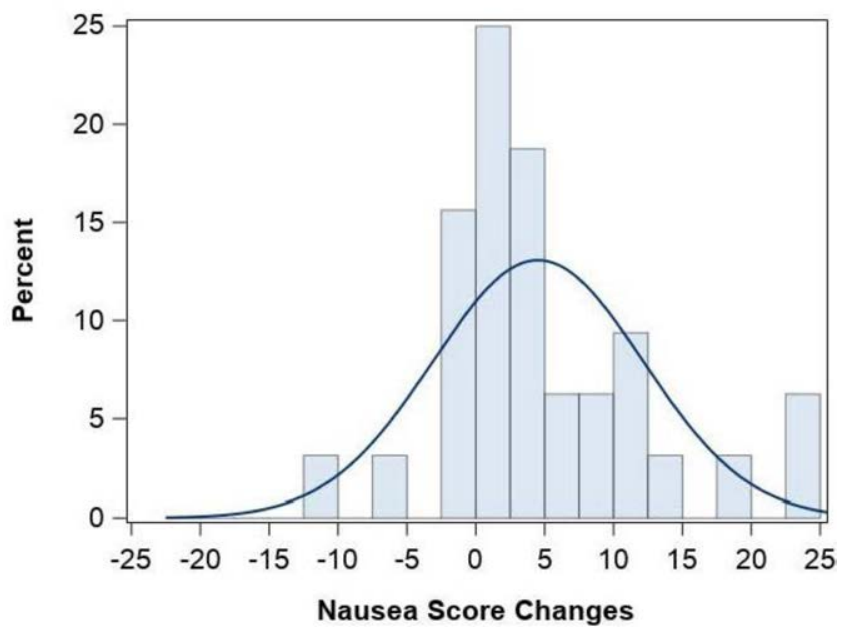


Figure 2. Fatigue PROMIS Score Changes Among Patients Reporting Fatigue as a Side Effect of MTX [ $n=96$ observations ( 48 pairs) among 28 unique pts]

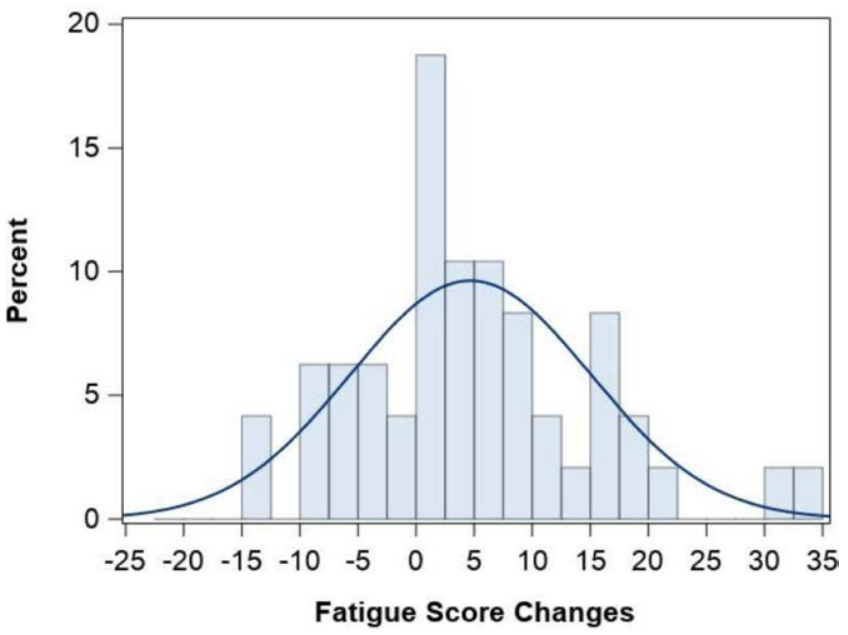

None declared, Hong Zhao: None declared, Fenglong Xie: None declared, Jeffrey Curtis Grant/research support from: AbbVie, Amgen, Bristol-Myers Squibb, Corrona, Janssen, Lilly, Myriad, Pfizer, Regeneron, Roche, UCB, Consultant of: AbbVie, Amgen, Bristol-Myers Squibb, Corrona, Janssen, Lilly, Myriad, Pfizer, Regeneron, Roche, UCB

DOI: 10.1136/annrheumdis-2020-eular.2354

\section{SAT0151 EFFICACY AND SAFETY OF UPADACITINIB VERSUS ABATACEPT IN PATIENTS WITH ACTIVE RHEUMATOID ARTHRITIS AND PRIOR INADEQUATE RESPONSE OR INTOLERANCE TO BIOLOGIC DISEASE-MODIFYING ANTI-RHEUMATIC DRUGS (SELECT-CHOICE): A DOUBLE-BLIND, RANDOMIZED CONTROLLED PHASE 3 TRIAL}

A. Rubbert-Roth ${ }^{1}$, J. Enejosa ${ }^{2}$, A. Pangan ${ }^{2}$, R. Xavier $^{3}$, B. Haraoui ${ }^{4}$ M. Rischmueller ${ }^{5}$, N. Khan ${ }^{2}$, Y. Zhang ${ }^{2}$, N. Martin ${ }^{2}$, M. C. Genovese ${ }^{6} .{ }^{1}$ Division of Rheumatology, Cantonal Clinic St Gallen, St Gallen, Switzerland; ${ }^{2}$ AbbVie Inc., North Chicago, United States of America; ${ }^{3}$ Universidade Federal do Rio Grande do Sul Porto Alegre, Rio Grande do Sul, Brazil; ${ }^{4}$ Centre hospitalier de I'Université de Montréal, Montréal, Canada; ${ }^{5}$ The Queen Elizabeth Hospital and University of Adelaide, Adelaide, Australia; ${ }^{6}$ Division of Immunology \& Rheumatology, Stanford University School of Medicine, Palo Alto, United States of America

Background: Upadacitinib (UPA) is an oral, reversible, selective JAK 1 inhibitor approved for the treatment of moderate to severe rheumatoid arthritis (RA). The efficacy/safety of UPA has been demonstrated in phase 3 studies, including superiority to adalimumab in patients (pts) with prior inadequate response (IR) to methotrexate..$^{1-4}$

Objectives: To assess the efficacy/safety of UPA vs abatacept (ABA) in pts with prior IR or intolerance to biologic DMARDs (bDMARDs).

Methods: Pts were randomized to once daily UPA $15 \mathrm{mg}$ or intravenous ABA (at Day 1, Weeks [Wks] 2, 4, 8, 12, 16 and 20 [< $60 \mathrm{~kg}: 500 \mathrm{mg} ; 60-100 \mathrm{~kg}: 750 \mathrm{mg}$; $>100 \mathrm{~kg}: 1,000 \mathrm{mg}]$ ), with all pts continuing background stable csDMARDs. The study was double-blind for 24 wks. Starting at Wk 12, pts who did not achieve $\geq 20 \%$ improvement from baseline (BL) in both tender and swollen joint counts at two consecutive visits, had background medication(s) adjusted or initiated. The primary endpoint was change from BL in DAS28(CRP) at Wk 12 (non-inferiority). The non-inferiority of UPA vs ABA was tested using the $95 \% \mathrm{Cl}$ of treatment difference against a non-inferiority margin of 0.6 . The two key secondary endpoints at Wk 12 were change from $\mathrm{BL}$ in DAS28(CRP) and the proportion of pts achieving clinical remission (CR) based on DAS28(CRP), defined as DAS28(CRP) <2.6. Both endpoints were to demonstrate the superiority of UPA vs. ABA. Treatment-emergent adverse events (TEAEs) are reported up to Wk 24 for all pts who received at least one dose of study drug.

Results: Of 612 pts treated; $67 \%$ of pts had received 1 prior bDMARD, $22 \%$ received 2 prior bDMARDs, and $10 \%$ received $\geq 3$ prior bDMARDs. $549(90 \%)$ completed 24 wks of treatment. Common reasons for study drug discontinuation were AEs (UPA, 3.6\%; ABA, 2.6\%) and withdrawal of consent (UPA, 1.7\%; ABA, $2.6 \%)$.

Non-inferiority and superiority were met for UPA vs ABA at Wk 12 for change from $B L$ in DAS28(CRP) (-2.52 vs $-2.00 ;-0.52[-0.69,-0.35]$; $p<0.001$ for UPA vs ABA).
UPA also demonstrated superiority to ABA in achieving DAS28(CRP) $<2.6(30.0 \%$ vs $13.3 \% ; p<0.001$ for UPA vs ABA; Figure 1). Improvements in disease activity and remission rates were maintained through Wk 24. The proportions of pts achieving low disease activity (defined as DAS28(CRP) $\leq 3.2$ ), ACR20, ACR50, and ACR70 responses were greater with UPA compared with ABA at Wk 12 (nominal $p<0.05$ ). More stringent outcome measures $-\mathrm{CR}$, ACR50, and ACR70 responses - remained higher with UPA than ABA through Wk 24 (nominal $p<0.05$ ). Incidence of serious TEAEs, AEs leading to discontinuation, hepatic disorders, and CPK elevations were numerically higher with UPA versus ABA (Figure 2). Eight cases of herpes zoster were reported (4 in each treatment arm). No malignancies were reported. One case of adjudicated MACE, two adjudicated cases of VTE ( $1 \mathrm{pt}$ with DVT and 1 pt with PE; both pts had at least one risk factor for VTE), and one treatment-emergent death were reported with UPA.

Conclusion: In RA pts with a prior IR or intolerance to bDMARDs, UPA demonstrated superior improvement in signs and symptoms vs ABA based on change in DAS28(CRP) and in achieving CR at Wk 12. The safety profile of UPA was consistent with the phase 3 RA studies with no new risks identified.

References:

[1] Burmester GR, et al. Lancet. 2018;391(10139):2503-12

[2] Fleischmann R, et al. Arthritis Rheumatol. 2019;71(11):1788-800

[3] Genovese MC, et al. Lancet. 2018;391(10139):2513-24

[4] Smolen JS, et al. Lancet. 2019;393(10188):2303-11

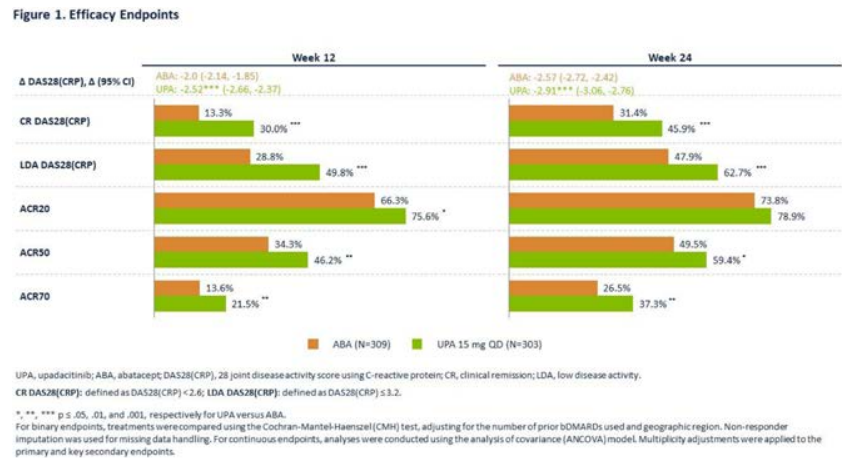

Figure 2. Safety Summary Through Week 24

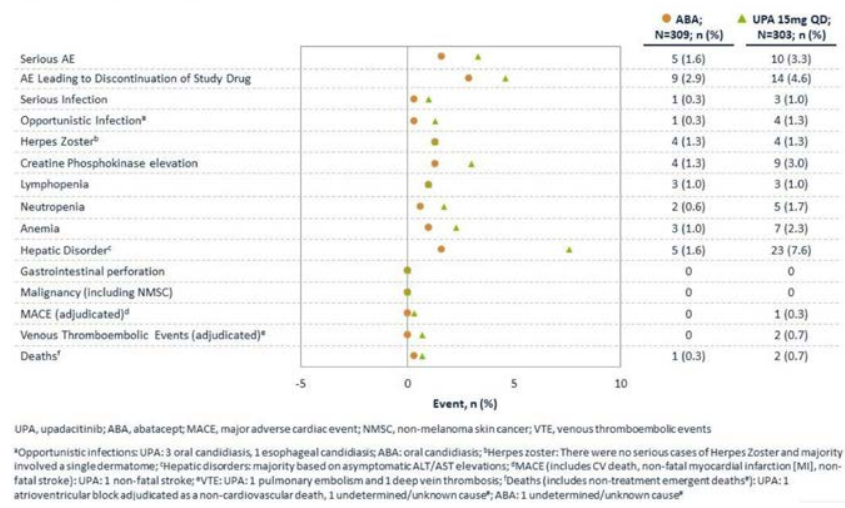

Disclosure of Interests: Andrea Rubbert-Roth Consultant of: Abbvie, BMS, Chugai, Pfizer, Roche, Janssen, Lilly, Sanofi, Amgen, Novartis, Jeffrey Enejosa Shareholder of: AbbVie Inc., Employee of: AbbVie Inc., Aileen Pangan Shareholder of: AbbVie Inc., Employee of: AbbVie Inc., Ricardo Xavier Consultant of: AbbVie, Pfizer, Novartis, Janssen, Eli Lilly, Roche, Boulos Haraoui Grant/ research support from: Abbvie, Amgen, Pfizer, UCB, Grant/research support from: AbbVie, Amgen, BMS, Janssen, Pfizer, Roche, and UCB, Consultant of: Abbvie, Amgen, Lilly, Pfizer, Sandoz, UCB, Consultant of: AbbVie, Amgen, BMS, Celgene, Eli Lilly, Janssen, Merck, Pfizer, Roche, and UCB, Speakers bureau: Pfizer, Speakers bureau: Amgen, BMS, Janssen, Pfizer, and UCB, Maureen Rischmueller Consultant of: Abbvie, Bristol-Meyer-Squibb, Celgene, Glaxo Smith Kline, Hospira, Janssen Cilag, MSD, Novartis, Pfizer, Roche, Sanofi, UCB, Nasser Khan Shareholder of: AbbVie Inc., Employee of: AbbVie Inc., Ying Zhang Shareholder of: AbbVie Inc., Employee of: AbbVie Inc., Naomi Martin Shareholder of: AbbVie Inc., Employee of: AbbVie Inc., Mark C. Genovese Grant/research support from: Abbvie, Eli Lilly and Company, EMD Merck Serono, Galapagos, Genentech/Roche, Gilead Sciences, Inc., GSK, Novartis, Pfizer Inc., RPharm, Sanofi Genzyme, Consultant of: Abbvie, Eli Lilly 